\title{
L'effet du trouble bipolaire I et du trouble dépressif majeur sur le fonctionnement de la main-d'œuvre
}

\author{
RS McIntyre, M.D., FRCPC (1,2,3); K Wilkins, M.Sc. (4); H Gilmour, M.A. (4); JK Soczynska, HBSc. (3,5); \\ JZ Konarksi, M.SC. (4); A Miranda (3); HO Woldeyohannes, HBSC. (3); D Vagic, M.D. (3); M Alsuwaidan, M.D. (1); \\ SH Kennedy, M.D., FRCPC $(1,3,5)$
}

\section{Résumé}

La présente enquête avait pour objet d'explorer et de comparer les effets du trouble bipolaire I (TB) et du trouble dépressif majeur (TDM) sur le fonctionnement de la maind'œuvre. Les données utilisées dans cette analyse sont tirées de l'Enquête sur la santé dans les collectivités canadiennes (ESCC, 1.2). L'échantillon était composé de 20747 sujets (de $\geq 18$ ans, détenant un emploi); les proportions de ceux chez qui le dépistage a établi la présence d'un TB et d'un TDM à vie étaient de 2,4 et 11,2\%, respectivement. Le revenu annuel moyen des sujets présentant un TB ou un TDM était significativement plus faible que celui des personnes qui en étaient exemptes. Par rapport aux sujets atteints d'un TDM, les personnes souffrant d'un TB avaient un revenu annuel significativement plus faible ( $\mathrm{p}<0,05)$. Si l'on se fie aux résultats d'une analyse de régression logistique multiple, les sujets atteints d'un TB étaient proportionnellement plus nombreux que les sujets atteints d'un TDM à avoir fait état d'au moins une journée d'incapacité pour des raisons de santé mentale au cours des deux dernières semaines ( $R C=1,6$; IC à $95 \%=1,0$ à 2,6). La probabilité de bénéficier d'une " bonne sécurité d'emploi "était plus faible chez les sujets atteints d'un TB que chez ceux souffrant d'un TDM (tous les sujets détenaient un emploi) ( $R C=0,6$; IC à $95 \%=0,5$ à 0,9). Les données présentées ici font ressortir l'effet pernicieux du TB sur le fonctionnement de la main-d'œuvre et indiquent qu'il y aurait lieu de soumettre toutes les personnes inscrites à un programme d'aide à l'emploi pour des raisons de dépression à un dépistage opportuniste du TB.

Mots clés : trouble bipolaire, trouble dépressif majeur, fonctionnement de la maind'ceuvre, capital humain

\section{Introduction}

Il existe de plus en plus de données indiquant que les troubles de l'humeur figurent parmi les principales causes d'incapacité et de décès prématuré(1,2) Selon des études sur le fardeau de la maladie, les coûts directs et indirects associés aux troubles de l'humeur dans les pays industrialisés et en développement sont renversants ${ }^{(3)}$. La diminution de la productivité auprès de la main-d'œuvre représente une composante importante du coût global de la maladie ${ }^{(4)}$. L'amélioration du dépistage et de la prise en charge des troubles de l'humeur contribuerait pour beaucoup à éviter les dépenses et à réduire les coûts afférents ${ }^{(5)}$.
La plupart des études sur le fardeau de la maladie sont fondées sur des sujets ayant reçu un diagnostic de trouble dépressif majeur (TDM), les études visant à évaluer séparément l'effet du trouble bipolaire (TB) étant relativement moins nombreuses ${ }^{(6-8)}$. Au cours de la dernière décennie, le taux estimatif de prévalence à vie du $\mathrm{TB}$ a considérablement augmenté, surtout en raison de l'élargissement des frontières diagnostiques $^{(9)}$. La part totale des coûts liés aux troubles de l'humeur attribuable au TB pourrait être considérablement plus élevée que ne l'indiquent les estimations antérieures ${ }^{(6,10)}$.

D’après la seule étude à présenter des données comparatives sur les coûts, auprès de la main-d'œuvre, du TDM et du TB, le TB était associé à un nombre plus élevé de jours de travail perdus par travailleur malade, par année ${ }^{(4)}$. Cet effet s'explique par la gravité et la persistance des symptômes dépressifs, qui sont plus grandes dans le cas du TB que dans le cas du TDM. L'étude indique également que le présentéisme (faible rendement au travail) comptait pour environ les deux tiers du coût total de la maladie pour la main-d'œuvre.

Cette enquête visait à décrire et à comparer l'effet du TB, par rapport au TDM et à l'état de santé de l'ensemble de

\footnotetext{
Coordonnées des auteurs

1 Département de psychiatrie, Université de Toronto, Toronto, ON, Canada

2 Département de pharmacologie, Université de Toronto, Toronto, ON, Canada

3 Mood Disorders Psychopharmacology Unit, University Health Network, Toronto, ON, Canada

4 Division de la statistique de la santé, Statistique Canada, Ottawa, ON, Canada

5 Institute of Medical Science, Université de Toronto, Toronto, ON, Canada

Correspondance : $\mathrm{D}^{r}$ Roger S. McIntyre, M.D., FRCPC, Professeur agrégé, départements de psychiatrie et de pharmacologie, Université de Toronto, Chef, Mood

Disorders Psychopharmacology Unit, University Health Network, 399 Bathurst Street, Toronto (ON), M5T 2S8, Tél. : (416) 603-5279, Téléc. : (416) 603-5368,

Courriel : roger.mcintyre@uhn.on.ca
} 
la population, sur le fonctionnement de la main d'œuvre. Cette analyse post hoc a été réalisée à la lumière des données d'une enquête transnationale relative à la santé mentale réalisée au Canada.

\section{Méthodologie}

Les données utilisées dans cette analyse proviennent de l'Enquête sur la santé dans les collectivités canadiennes : santé mentale et bien-être (82-617-XIF), menée en 2002 par Statistique Canada dans le cadre de l'ESCC. Des renseignements sur l'enquête sont disponibles à l'adresse http://www.statcan.ca: $8096 /$ bsolc/ francais/bsolc?catno $=82-617-X$. L'enquête a fait appel à la version du Composite International Diagnostic Interview (WHSCIDI) utilisée par l'Organisation mondiale de la santé (OMS), qui fait partie de l'initiative "World Mental Health 2000 ». On peut trouver le questionnaire utilisé à l'adresse : http://www.statcan.ca/francais/ sdds/instrument/5015_Q1_V1_F.pdf/.

Les répondants étaient des personnes vivant dans des logements privés, lesquels ont été choisis à la lumière d'un plan d'échantillonnage en grappes stratifié à plusieurs degrés. La plupart des interviews (86 \%) ont été réalisées en personne; le reste par téléphone. L'échantillon de répondants était composé en tout de 36984 personnes âgées de 15 ans ou plus; le taux de participation a été élevé (77\%). Les données ont été pondérées pour qu'elles soient représentatives de la population à domicile des dix provinces du Canada en 2002. L'analyse ne concernait que des sujets de 18 ans et plus, détenant un emploi.

L'ESCC a recueilli des éléments d'information sur les déterminants et les corrélats de la santé mentale, comme le profil sociodémographique, le revenu, la détresse autodéclarée, le niveau d'activité physique durant les loisirs, la consommation de médicaments et le soutien social. À partir des critères de dépistage définis par le WHS-CIDI, l'enquête a recueilli des données sur la prévalence à vie et au cours des 12 derniers mois de divers troubles mentaux et comportements connexes (soit épisode dépressif majeur, épisode maniaque, trouble panique, agoraphobie, phobie sociale, dépendance à l'égard de l'alcool et des drogues, jeu pathologique, suicide et comportement alimentaire anormal), la taille et le poids autodéclarés et les antécédents de problèmes de santé diagnostiqués. Les variables liées au travail comprenaient la situation d'emploi actuelle, l'autoperception de la sécurité d'emploi et le nombre de " jours d'incapacité pour des raisons de santé mentale » déclarés au cours des deux dernières semaines. Afin de mesurer cette dernière variable, on a demandé aux répondants si, au cours des deux dernières semaines, ils avaient dû garder le lit pendant toute une journée ou presque ou limiter leurs activités habituelles à cause d'une maladie ou d'une blessure, ou encore, s'ils avaient dû faire plus d'effort pour maintenir leur niveau habituel de fonctionnement au travail ou dans leurs activités quotidiennes à cause de leur santé émotionnelle ou mentale ou de leur consommation d'alcool ou de drogues.

Les analyses statistiques ont été réalisées à l'aide de calculs de fréquences, de tableaux à entrées multiples et de modèles de régression multiples. Toutes les analyses étaient fondées sur des données qui ont été pondérées pour qu'elles soient représentatives de la population canadienne (âgée de 18 ans ou plus) de 2002. À partir des données concernant des personnes déclarées positives à l'égard d'un trouble de l'humeur (trouble bipolaire I ou dépression) à vie à la suite d'un dépistage, on a fait appel à des modèles de régression logistique multiples pour comparer les associations entre le trouble bipolaire Ià vie et le trouble dépressif à vie et, d'une part, l'autodéclaration de la sécurité d'emploi et, d'autre part, les jours d'incapacité pour des raisons de santé mentale déclarés au cours des deux dernières semaines. Pour les besoins de l'analyse, on a créé des groupes mutuellement exclusifs : sujets déclarés atteints d'un trouble bipolaire I à vie (TB) à la suite d'un dépistage; sujets déclarés atteints d'un trouble dépressif majeur à vie (sans trouble bipolaire I) (TDM) à la suite d'un dépistage et sujets ne souffrant d'aucun de ces deux troubles de l'humeur.
Les modèles tenaient compte des effets du sexe, du groupe d'âge, du niveau de scolarité, de la présence d'une affection chronique diagnostiquée par un médecin (arthrite, asthme, problèmes de dos sauf la fibromyalgie et l'arthrite, hypertension artérielle, migraine, bronchite chronique, emphysème ou maladie pulmonaire obstructive chronique, diabète, épilepsie, cardiopathie, cancer, ulcères gastriques ou intestinaux, effets d'un accident vasculaire cérébral, troubles intestinaux/maladie de Crohn ou colite, maladie d'Alzheimer ou autre démence, cataractes, glaucome et dérèglement thyroïdien) et de l'indice de masse corporelle. L'analyse préliminaire des données a fait ressortir un taux relativement élevé de prévalence de la dépendance à une substance (alcool ou drogues illicites) chez les personnes atteintes d'un TB ou d'un TDM. Les taux estimatifs de dépendance au cours des douze mois précédents s'élevaient à 23 et à $7 \%$, respectivement, contre $3 \%$ dans l'ensemble de la population (données non présentées). C'est pourquoi, afin d'éviter la multicollinéarité, aucune variable relative à la dépendance à une substance n'a été incluse dans les modèles de régression multidimensionnelles.

Toutes les analyses statistiques ont été réalisées à l'aide du logiciel statistique SAS, version 9.1 (SAS Institute, Cary, Caroline du Nord). Les variables retenues ont été présélectionnées à la lumière de recherches antérieures. Compte tenu de la complexité du plan d'échantillonnage de l'ESCC, on a fait appel à la technique d'amorce pour calculer les coefficients de variation des estimations et l'importance des écarts entre les estimations. Le niveau de signification statistique a été fixé à $p<0,05$.

\section{Résultats}

Un taux estimatif de 2,4\% de la population canadienne active de 18 ans ou plus avait eu un épisode maniaque à vie (c.-à-d. un trouble bipolaire I, selon la définition du DSM-IV). Des antécédents de TB à vie étaient plus fréquents chez les répondants plus jeunes, les personnes non mariées ou 
ne vivant pas avec un partenaire, et celles qui n'avaient pas terminé des études postsecondaires (tableau 1). La proportion de la population active de 18 ans ou plus qui a été déclarée positive à l'égard du TDM (sans TB) à vie à la suite d'un dépistage a été estimée à $11,2 \%$

Parmi les Canadiens de 18 ans ou plus en 2002, la proportion de ceux qui détenaient un emploi ne différait pas de manière significative en fonction de la présence d'un trouble de l'humeur à vie. On n'a pas observé non plus de différence significative entre la proportion atteinte d'un TB et celle souffrant d'un TDM à vie. Chez les personnes sans antécédent de trouble de l'humeur, $70 \%$ détenaient un emploi; la proportion de sujets qui ont déclaré avoir un emploi au moment de l'enquête était de $68 \%$ chez ceux qui présentaient un TB et de $69 \%$ chez ceux qui souffraient d'un TDM (données non présentées). Dans les trois groupes, la plupart des sujets qui étaient actifs détenaient un emploi à plein temps (86\% chez ceux qui ne souffraient d'aucun trouble de l'humeur; $87 \%$ chez ceux présentant un TB à vie, $84 \%$ chez ceux présentant un TDM à vie; les différences entre les proportions

TABLEAU 1

Prévalence à vie du trouble bipolaire I (TB) et du trouble dépressif majeur (TDM) (sans TB), selon certaines caractéristiques sociodémographiques, population active à domicile de 18 ans ou plus, Canada (à l'exclusion des territoires), 2002

\begin{tabular}{|c|c|c|c|c|c|c|}
\hline & \multicolumn{2}{|c|}{ TB à vie } & \multicolumn{2}{|c|}{ TDM (sans TB) à vie } & \multicolumn{2}{|c|}{ Ni TB ni TDM à vie } \\
\hline & $\begin{array}{l}\text { Nombre } \\
\text { estimatif }\end{array}$ & $\%$ & $\begin{array}{l}\text { Nombre } \\
\text { estimatif }\end{array}$ & $\%$ & $\begin{array}{l}\text { Nombre } \\
\text { estimatif }\end{array}$ & $\%$ \\
\hline \multicolumn{7}{|l|}{ Sexe } \\
\hline Hommes $^{\dagger}$ & 207000 & 2,5 & 677000 & 8,1 & 7506000 & 89,5 \\
\hline 18 à 34 ans & 146000 & $2,8^{*}$ & 529000 & $10,0^{*}$ & 4592000 & 87,2 \\
\hline 35 ans et plus ${ }^{\dagger}$ & 225000 & 2,2 & 1208000 & 11,9 & 8728000 & 85,9 \\
\hline \multicolumn{7}{|l|}{ Niveau de scolarité } \\
\hline Diplôme d'études secondaires ou moins & 140000 & 2,6 & 512000 & $9,7^{*}$ & 4628000 & 87,6 \\
\hline Marié(e)/vivant avec conjoint ${ }^{\dagger}$ & 177000 & 1,7 & 1503000 & 10,1 & 9195000 & 88,2 \\
\hline Séparé(e)/divorcé(e)/veuf ou veuve & 77000 & $5,7^{*}$ & 293000 & $21,4^{*}$ & 997000 & $72,9^{*}$ \\
\hline Jamais marié(e) & 117000 & $3,2^{*}$ & 390000 & 10,8 & 3110000 & 86,0 \\
\hline
\end{tabular}

${ }^{\dagger}$ Groupe de référence

* L'estimation est significativement différente de celle qui s'applique au groupe de référence $(p<0,05)$.

Étant donné que les chiffres ont été arrondis et que certaines données manquent (niveau de scolarité), leur somme ne correspond pas nécessairement aux totaux indiqués.

Source : Enquête sur la santé dans les collectivités canadiennes : santé mentale et bien-être, 2002

TABLEAU 2

Revenu annuel moyen, selon la présence d'un trouble de l'humeur à vie (trouble bipolaire I [TDM], population active à domicile de 18 ans ou plus, Canada (à l'exclusion des territoires), 2002

\begin{tabular}{lc}
\hline Trouble & Revenu moyen (\$ CA) \\
\hline TB à vie & $32000^{*}$ \\
TDM (sans TB) à vie & 36800 \\
Population générale exemple de trouble de l’humeur & 40300 \\
\hline & * L'estimation est significativement différente de celle qui s'applique au groupe de référence $(p<0,05)$. \\
Source : Enquête sur la santé dans les collectivités canadiennes : santé mentale et bien-être, 2002
\end{tabular}


TABLEAU 3a

Rapports de cotes corrigés associés à une incapacité d'au moins un jour pour des raisons de santé mentale au cours des deux dernières semaines, relatifs à la présence d'un trouble de l'humeur (trouble bipolaire I [TB] à vie ou trouble dépressif majeur [TDM] à vie), selon certaines caractéristiques, population active à domicile de 18 ans ou plus, Canada (exclusion faite des territoires), 2002

\begin{tabular}{|c|c|c|}
\hline & Rapport de cotes & $\begin{array}{c}\text { Intervalle de } \\
\text { confiance à } 95 \%\end{array}$ \\
\hline \multicolumn{3}{|l|}{ Trouble de I'humeur } \\
\hline TB à vie & $8,8^{*}$ & 5,9 à 13,1 \\
\hline TDM à vie (sans TB) & $5,6^{*}$ & 4,1 à 7,7 \\
\hline Aucun ${ }^{\dagger}$ & 1,0 & - \\
\hline \multicolumn{3}{|l|}{ Sexe } \\
\hline Hommes & 0,8 & 0,6 à 1,0 \\
\hline Femmes $^{\dagger}$ & 1,0 & - \\
\hline \multicolumn{3}{|l|}{ Groupe d'âge } \\
\hline 18 à 34 ans & $1,6^{*}$ & 1,2 à 2,1 \\
\hline 35 ans et plus ${ }^{\dagger}$ & 1,0 & - \\
\hline \multicolumn{3}{|l|}{ Niveau de scolarité } \\
\hline Diplôme d'études secondaires ou moins ${ }^{\dagger}$ & 1,0 & - \\
\hline Cetaines études post-secondaires & 0,8 & 0,5 à 1,3 \\
\hline Diplôme d'études post-secondaires & $0,7^{*}$ & 0,5 à 1,0 \\
\hline \multicolumn{3}{|l|}{ Comorbidité } \\
\hline Présence d'une maladie chronique ${ }^{\S}$ & $2,0^{*}$ & 1,5 à 2,8 \\
\hline \multicolumn{3}{|l|}{ Catégorie relative à l'indice de masse corporelle } \\
\hline $\begin{array}{l}\text { Poids insuffisant/poids normal/surpoids } \\
(\text { IMC }<30,0)^{\dagger}\end{array}$ & 1,0 & - \\
\hline Obésité (IMC $\geq 30,0$ ) & 0,8 & 0,5 à 1,1 \\
\hline
\end{tabular}

* L'éstimation est significativement différente de celle qui s'applique au groupe de référence $(p<0,05)$.

† Groupe de référence, pour lequel le rapport de cotes est toujours de 1,0

- Sans objet

$\S$ Le groupe de référence est exempt des affections suivantes : arthrite, asthme, problèmes de dos sauf la fibromyalgie et l'arthrite, hypertension artérielle, migraine, bronchite chronique, emphysème ou maladie pulmonaire obstructive chronique, diabète, épilepsie, cardiopathie, cancer, ulcères gastriques ou intestinaux, effets d'un accident vasculaire cérébral, trouble intestinaux/maladie de Crohn ou colite, maladie d'Alzheimer ou autre démence, cataractes, glaucome et dérèglement thyrö̈dien.

Les rapports de cotes ont été arrondis, certaines dont la limite de confiance inférieure ou supérieure est de 1,0 étant statistiquement significatifs (comme il est indiqué).

Le modèle est fondé sur des enregistrements relatifs à 20431 répondants.

Source : Enquête sur la santé dans les collectivités canadiennes : santé mentale et bien-être 2002 n’étaient pas statistiquement significatives) (données non présentées).

Le revenu annuel moyen des personnes détenant un emploi et atteintes d'un trouble de l'humeur était sensiblement plus faible que celui des personnes qui en étaient exemptes. Il était de 32000 \$ chez les sujets présentant un TB à vie, soit près de 5000 \$ de moins que le revenu moyen (36 800 \$) des sujets atteints d'un TDM $(p<0,05)$ (tableau 2). Chez les personnes ne souffrant d'aucun des deux troubles de l'humeur, le revenu annuel moyen s'élevait à 40300 \$, ce qui est sensiblement et statistiquement plus élevé que celui de l'un ou l'autre des groupes atteints d'un trouble de l'humeur $(p<0,05)$.

Selon les résultats obtenus avec les modèles multidimensionnels, la probabilité d'avoir fait état d'au moins une journée d'incapacité pour des raisons de santé mentale au cours des deux dernières semaines était significativement plus élevée chez les per-sonnes présentant un TB à vie $(\mathrm{RC}=8,8$; IC à $95 \%=5,9$ à 13,1$)$ ou un TDM à vie $(\mathrm{RC}=5,6$; IC à $95 \%=4,1$ à 7,7 ), que chez les sujets ne présentant aucun de ces troubles (tableau 3a). La probabilité d'avoir connu au moins une journée d'incapacité pour des raisons de santé mentale au cours des deux dernières semaines était significativement plus élevée chez les personnes présentant un TB à vie que chez celles présentant un $\mathrm{TDM}$ à vie $(\mathrm{RC}=1,6$; $\mathrm{IC}$ à $95 \%=1,0$ à 2,6) (tableau 3b). De même, la probabilité de déclarer jouir d'une bonne sécurité d'emploi était significativement plus faible chez les personnes atteintes d'un TB $(\mathrm{RC}=0,4 ; \mathrm{IC}$ à $95 \%=0,3$ à 0,6$)$ ou d'un $\mathrm{TDM}(\mathrm{RC}=0,7$; IC à $95 \%=0,6$ à 0,8$)$ que chez celles ne présentant aucun de 
ces troubles de l'humeur (tableau 4a). Par ailleurs, sur ce plan, les personnes atteintes d'un TB (RC $=0,6$; IC à $95 \%=0,5$ à 0,9$)$ étaient désavantagées par rapport à celles qui présentaient un TDM (tableau 4b). Ces associations étaient présentes après la prise en compte de l'effet de possibles facteurs confusionnels : sexe, groupe d'âge, niveau de scolarité, comorbidité et indice de masse corporelle.

\section{Analyse}

Si l'on se fie à ces résultats, d'après plusieurs indices du fonctionnement de la main-d'œuvre, les incapacités sont nettement plus fréquentes chez les sujets atteints soit d'un TB soit d'un TDM que celles qui ne présentent aucun trouble de l'humeur. Ces données indiquent en outre que les incapacités sont sans doute plus fréquentes chez les sujets atteints d'un TB que chez ceux souffrant d'un TDM, comme en témoignent le revenu annuel, le nombre de jours d'incapacité pour des raisons de santé mentale et la sécurité d’emploi.

La seule autre enquête à présenter des données comparatives sur les coûts, pour la main-d'œuvre, associés au TDM et au TB a aussi révélé que les troubles de l'humeur étaient un facteur de prédiction d'une perte globale de rendement au travail, le nombre estimatif de jours de travail perdus par travailleur étant de 65,5 dans le cas du TB et de 27,2 dans le cas du TDM(4). Aucun écart significatif n'a été observé dans l'association entre le TB ou le TDM et le rendement au travail, en fonction du sexe ou de l'âge. La perte de travail associée au TB a touché tous les groupes professionnels, l'effet étant significativement plus marqué chez les techniciens et les professionnels
TABLEAU 3b

Rapports de cotes corrigés associés à une incapacité pour des raisons de santé mentale au cours des deux dernières semaines, en rapport avec la présence d'un trouble bipolaire I (TB) à vie ou d'un trouble dépressif majeur (TDM) à vie, selon certaines caractéristiques, population active à domicile de 18 ans ou plus souffrant d'un trouble de l'humeur, Canada (exclusion faite des territoires), 2002

\begin{tabular}{|c|c|c|}
\hline & Rapport de cotes & $\begin{array}{c}\text { Intervalle de } \\
\text { confiance à } 95 \%\end{array}$ \\
\hline \multicolumn{3}{|l|}{ Trouble de l'humeur } \\
\hline TB à vie & $1,6^{*}$ & 1,0 à 2,6 \\
\hline TDM à vie (sans TB) & 1,0 & - \\
\hline \multicolumn{3}{|l|}{ Sexe } \\
\hline Hommes & 0,9 & 0,5 à 1,4 \\
\hline Femmes $^{\dagger}$ & 1,0 & - \\
\hline \multicolumn{3}{|l|}{ Groupe d'âge } \\
\hline 18 à 34 ans & 1,5 & 0,9 à 2,5 \\
\hline 35 ans et plus & 1,0 & - \\
\hline \multicolumn{3}{|l|}{ Niveau de scolarité } \\
\hline Diplôme d'études secondaires ou moins ${ }^{\dagger}$ & 1,0 & - \\
\hline Certaines études post-secondaires & $0,5^{*}$ & 0,2 à 1,0 \\
\hline Diplôme d'études post-secondaires & $0,5^{*}$ & 0,3 à 0,8 \\
\hline \multicolumn{3}{|l|}{ Comorbidité } \\
\hline Présence d'une maladie chronique ${ }^{\S}$ & $2,0^{*}$ & 1,2 à 3,3 \\
\hline \multicolumn{3}{|l|}{ Catégorie relative à l'indice de masse corporelle } \\
\hline $\begin{array}{l}\text { Poids insuffisant/poids normal/surpoids } \\
(\mathrm{IMC}<30,0)^{\dagger}\end{array}$ & 1,0 & - \\
\hline Obésité (IMC $\geq 30,0$ ) & 1,2 & 0,7 à 1,9 \\
\hline
\end{tabular}

* L'estimation est significativement différente de celle qui s'applique au groupe de référence $(p<0,05)$.

+ Groupe de référence, pour lequel le rapport de cotes est toujours de 1,0

- Sans objet

${ }^{\S}$ Le groupe de référence est exempt des affections suivantes : arthrite, asthme, problèmes de dos sauf la fibromyalgie et l'arthrite, hypertension artérielle, migraine, bronchite chronique, emphysème ou maladie pulmonaire obstructive chronique, diabète, épilepsie, cardiopathie, cancer, ulcères gastriques ou intestinaux, effets d'un accident vasculaire cérébral, trouble intestinaux/maladie de Crohn ou colite, maladie d'Alzheimer ou autre démence, cataractes, glaucome et dérèglement thyroïdien.

Les rapports de cotes ont été arrondis, certaines dont la limite de confiance inférieure ou supérieure est de 1,0 étant statistiquement significatifs (comme il est indiqué).

Le modèle est fondé sur des enregistrements relatifs à 2999 répondants chez qui le dépistage a établi la présence d'un TDM ou d'un TB à vie.

Source : Enquête sur la santé dans les collectivités canadiennes : santé mentale de bien-être, 2002 
TABLEAU 4a

Rapports de cotes corrigés associés à une bonne sécurité d'emploie, en rapport avec la présence d'un trouble de l'humeur (trouble bipolaire I [TB] à vie ou trouble dépressif majeur [TDM] à vie), en fonction de certaines caractéristiques, population active à domicile de 18 ans ou plus, Canada, à l'exclusion des territoires, 2002

\begin{tabular}{|c|c|c|}
\hline & Rapport de cotes & $\begin{array}{c}\text { Intervalle de } \\
\text { confiance à } 95 \%\end{array}$ \\
\hline \multicolumn{3}{|l|}{ Trouble de l'humeur } \\
\hline TB à vie & $0,4^{*}$ & 0,3 à 0,6 \\
\hline TDM à vie (sans TB) & $0,7^{*}$ & 0,6 à 0,8 \\
\hline Aucun ${ }^{\dagger}$ & 1,0 & - \\
\hline \multicolumn{3}{|l|}{ Sexe } \\
\hline Hommes & 1,0 & 0,9 à 1,1 \\
\hline Femmes $^{\dagger}$ & 1,0 & - \\
\hline \multicolumn{3}{|l|}{ Groupe d'âge } \\
\hline 18 à 34 ans & 1,0 & 0,9 à 1,1 \\
\hline 35 ans et plus ${ }^{\dagger}$ & 1,0 & - \\
\hline \multicolumn{3}{|l|}{ Niveau de scolarité } \\
\hline Diplôme d'études secondaires ou moins ${ }^{\dagger}$ & 1,0 & - \\
\hline Certaines études post-secondaires & 0,9 & 0,8 à 1,1 \\
\hline Diplôme d'études post-secondaires & $0,9^{*}$ & 0,8 à 1,0 \\
\hline \multicolumn{3}{|l|}{ Comorbidité } \\
\hline Présence d'une maladie chronique ${ }^{\S}$ & 0,9 & 0,8 à 1,0 \\
\hline \multicolumn{3}{|l|}{ Catégorie relative à l'indice de masse corporelle } \\
\hline $\begin{array}{l}\text { Poids insuffisant/poids normal/surpoids } \\
(\mathrm{IMC}<30,0)^{\dagger}\end{array}$ & 1,0 & - \\
\hline Obésité (IMC $\geq 30,0$ ) & 1,0 & 0,9 à 1,2 \\
\hline
\end{tabular}

* L'estimation est significativement différente de celle qui s'applique au groupe de référence $(p<0,05)$.

† Groupe de référence, pour lequel le rapport de cotes est toujours de 1,0

- Sans objet

${ }^{\S}$ Le groupe de référence est exempt des affections suivantes : arthrite, asthme, problèmes de dos sauf la fibromyalgie et l'arthrite, hypertension artérielle, migraine, bronchite chronique, emphysème ou maladie pulmonaire obstructive chronique, diabète, épilepsie, cardiopathie, cancer, ulcères gastriques ou intestinaux, effets d'un accident vasculaire cérébral, trouble intestinaux/maladie de Crohn ou colite, maladie d'Alzheimer ou autre démence, cataractes, glaucome et dérèglement thyroïdien.

Les rapports de cotes ont été arrondis, certaines dont la limite de confiance inférieure ou supérieure est de 1,0 étant statistiquement significatifs (comme il est indiqué).

Le modèle est fondé sur des enregistrements relatifs à 20251 répondants.

Source : Enquête sur la santé dans les collectivités canadiennes : santé mentale de bien-être, 2002 en ce qui a trait à l'absentéisme, et chez les ouvriers et les professionnels, dans le cas du présentéisme.

Plusieurs données recueillies au cours de la dernière décennie sur les troubles de l'humeur permettent de bien situer ce phénomène dans les études sur les problèmes de fonctionnement de la maind'œuvre. Premièrement, le taux estimatif de prévalence à vie d'un trouble bipolaire est considérablement plus élevé que ne l'indiquaient les estimations antérieures ${ }^{(9)}$. Ainsi, on estime maintenant que de 2 à $5 \%$ de l'ensemble de la population pourrait être atteinte d'un $\mathrm{TB}^{(11,12)}$. Dans un tel contexte, il est possible que le pourcentage de personnes atteintes d'un TB, aptes au travail, soit plus élevé que ne le laissent entendre les estimations antérieures. Deuxièmement, d'après la structure symptomatique longitudinale du TB, qui s'apparente à celle du TDM, ce sont les symptômes dépressifs soussyndromiques chroniques qui dominent (13-16). Il est de plus en plus reconnu que, dans le TB, les symptômes dépressifs sont plus incapacitants que les symptômes maniaques ${ }^{(17)}$. De plus, les personnes souffrant de troubles du spectre bipolaire (un phénotype essentiellement dépressif) se situent entre les personnes atteintes d'un trouble bipolaire I et les non-cas qui font partie de l'ensemble de la population ${ }^{(9,18)}$. Troisièmement, selon plusieurs études sur le coût de la maladie, les troubles de l'humeur sont associés à une baisse sensible du rendement au travail, à un fardeau pour les soignants, à l'utilisation de services médicaux et à des décès prématurés ${ }^{(1,7,19)}$. Il a été prouvé que l'amélioration de la détection et de la prise en charge de la dépression autre que bipolaire entraînait 
une réduction du fardeau global de la maladie et des coûts afférents ${ }^{(20)}$.

Plusieurs facteurs viennent restreindre les interprétations que l'on peut donner aux données de cette analyse et les conclusions que l'on peut en tirer, le principal étant que l'ESCC n'a pas été conçue au départ pour évaluer et comparer les effets des troubles de l'humeur sur le fonctionnement de la main d'œuvre. L'ESCC ne comportait pas de mesure quantitative du rendement au travail (p. ex. le World Health Organization Health and Work Performance Questionnaire). De plus, nous n'avons pas pu nous prononcer sur les composantes des problèmes de fonctionnement et sur leur contribution au rendement et aux coûts globaux. Ainsi, il est possible que le présentéisme représente une part plus importante des coûts pour la main-d'œuvre, par rapport à l'absentéisme chez les personnes atteintes d'un $\mathrm{TB}^{(4)}$. Dans cette perspective, l'ESCC ne contient pas de mesures longitudinales détaillées, ce qui nous empêche de commenter la relative contribution des symptômes dépressifs (ou maniaques) au rendement global au travail. Il ressort d'une analyse des symptômes contenue dans l'étude comparative réalisée par Kessler et coll. que l'effet plus important du TB sur le rendement au travail s'explique par la gravité et la persistance accrues des symptômes dépressifs chez les sujets atteints d'un TB. Un autre facteur limite l'interprétation que l'on peut donner aux résultats de l'analyse, à savoir que l'échantillon de l'étude ne comprend que des sujets détenant un emploi. Plus précisément, toute tendance selon laquelle une proportion exagérée de personnes atteintes d'un TB ou d'un TDM aurait cessé de faire partie de la population active (ou n'en
TABLEAU $4 \mathrm{~b}$

Rapports de cotes corrigés associés à une bonne sécurité d'emploi, en rapport avec la présence d'un trouble bipolaire I (TB) à vie ou d'un trouble dépressif majeur (TDM) à vie, selon certaines caractéristiques, population active à domicile de $\mathbf{1 8}$ ans ou plus souffrant d'un trouble de l'humeur, Canada (à l'exclusion des territoires), 2002

\begin{tabular}{|c|c|c|}
\hline & Rapport de cotes & $\begin{array}{c}\text { Intervalle de } \\
\text { confiance à } 95 \%\end{array}$ \\
\hline \multicolumn{3}{|l|}{ Trouble de l'humeur } \\
\hline TB à vie & $0,6^{*}$ & 0,5 à 0,9 \\
\hline TDM à vie' (sans TB) & 1,0 & - \\
\hline \multicolumn{3}{|l|}{ Sexe } \\
\hline Hommes & $0,8^{*}$ & 0,6 à 1,0 \\
\hline Femmes $^{\dagger}$ & 1,0 & - \\
\hline \multicolumn{3}{|l|}{ Groupe d'âge } \\
\hline 18 à 34 ans & 0,9 & 0,7 à 1,1 \\
\hline 35 ans et plus ${ }^{\dagger}$ & 1,0 & - \\
\hline \multicolumn{3}{|l|}{ Niveau de scolarité } \\
\hline Diplôme d'études secondaires ou moins ${ }^{\dagger}$ & 1,0 & - \\
\hline Certaines études post-secondaires & $0,6^{*}$ & 0,4 à 0,9 \\
\hline Diplôme d'études post-secondaires & 0,8 & 0,6 à 1,1 \\
\hline \multicolumn{3}{|l|}{ Comorbidité } \\
\hline Présence d'une maladie chronique ${ }^{\S}$ & 0,9 & 0,7 à 1,2 \\
\hline \multicolumn{3}{|l|}{ Catégorie relative à l'indice de masse corporelle } \\
\hline $\begin{array}{l}\text { Poids insuffisant/poids normal/surpoids } \\
(\mathrm{IMC}<30,0)^{+}\end{array}$ & 1,0 & - \\
\hline Obésité (IMC $\geq 30,0)$ & 0,8 & 0,6 à 1,2 \\
\hline
\end{tabular}

* L'estimation est significativement différente de celle qui s'applique au groupe de référence $(p<0,05)$.

${ }^{+}$Groupe de référence, pour lequel le rapport de cotes est toujours de 1,0

- Sans objet

${ }^{\S}$ Le groupe de référence est exempt des affections suivantes : arthrite, asthme, problèmes de dos sauf la fibromyalgie et l'arthrite, hypertension artérielle, migraine, bronchite chronique, emphysème ou maladie pulmonaire obstructive chronique, diabète, épilepsie, cardiopathie, cancer, ulcères gastriques ou intestinaux, effets d'un accident vasculaire cérébral, trouble intestinaux/maladie de Crohn ou colite, maladie d'Alzheimer ou autre démence, cataractes, glaucome et dérèglement thyroïdien.

Les rapports de cotes ont été arrondis, certaines dont la limite de confiance inférieure ou supérieure est de 1,0 étant statistiquement significatifs (comme il est indiqué).

Le modèle est fondé sur des enregistrements relatifs à 2966 répondants chez qui le dépistage a établi la présence d'un TDM ou d'un TB à vie.

Source : Enquête sur la santé dans les collectivités canadiennes : santé mentale de bien-être, 2002 
aurait jamais fait partie), par rapport aux personnes exemptes de ces troubles, aurait pour effet de diluer la force de l'association observée entre le trouble de l'humeur et les problèmes de fonctionnement au travail.

L'ESCC comporte d'autres limites : le diagnostic était fondé sur le CIDI, qui est un instrument moins utile pour distinguer les épisodes mixtes et les troubles du spectre bipolaire du $\mathrm{TDM}^{(21)}$; le manque de données sur les antécédents de travail et le manque de détails sur les effets observés dans les différents groupes professionnels; l'absence de mesures objectives du rendement au travail; le recours aux données autodéclarées pour toutes les variables dépendantes; l'inclusion de sujets dont les épisodes maniaques pourraient avoir été causés par l'abus de substances; l'incapacité de tenir compte de l'effet du traitement. De plus, bien que les versions antérieures du CIDI aient été validées pour une utilisation lors d'enquêtes menées dans la communauté, l'ESCC a fait appel, dans le cadre de l'initiative 2000 World Mental Health, à une version actualisée de cet outil, laquelle n'a pas encore été entièrement validée. C'est pourquoi on ignore dans quelle mesure des évaluations faites par des cliniciens dûment formés rejoindraient les conclusions de l'ESCC. Quoi qu'il en soit, les sujets chez qui le dépistage avait donné un résultat positif à l'égard du TDM ou du TB dans l'ESCC sont représentatifs d'autres personnes atteintes de ces troubles sur les plans suivants : prévalence à vie, profil démographique, comorbidité, utilisation de services médicaux et résultats sur le plan fonctionnel ${ }^{(22-24)}$.

De manière générale, on sait que les effets des troubles de l'humeur sur les travailleurs touchés varient d'un trouble à l'autre ${ }^{(3)}$. Étant donné la plus forte prévalence du TDM dans l'ensemble de la population (et auprès de la main-d'œuvre), par rapport au TB, le coût global du TDM est plus élevé( $^{(4)}$. Quoi qu'il en soit, à une échelle individuelle, la présente étude et l'enquête réalisée par Kessler et coll. portent à croire que les coûts associés au TB pourraient être plus élevés. De là l'idée qu'il est peutêtre plus rentable de mettre en œuvre des programmes de dépistage et d'intervention visant le trouble bipolaire I et les troubles du spectre bipolaire que des programmes visant le $\operatorname{TDM}^{(8)}$. Il y aurait lieu d'intégrer le dépistage rapide et opportuniste du TB aux programmes de santé comportementale et d'aide à l'emploi.

\section{Références}

1. Greenberg PE, Stiglin LE, Finkelstein SN, et coll. The economic burden of depression in 1990. J Clin Psychiatry 1993 November;54(11):405-18.

2. McIntyre RS, Konarski JZ. Bipolar disorder: a national health concern. CNS Spectr 2004 December;9(11 Suppl 12):6-15.

3. Murray CJ, Lopez AD. Global mortality, disability, and the contribution of risk factors: Global Burden of Disease Study. Lancet 1997 May 17;349(9063):1436-42.

4. Kessler RC, Akiskal HS, Ames M, et coll. Prevalence and effects of mood disorders on work performance in a nationally representative sample of U.S. workers. Am J Psychiatry 2006 September;163(9):1561-8.

5. Kennedy SH, Lam RW, Cohen NL, et coll. Clinical guidelines for the treatment of depressive disorders. IV. Medications and other biological treatments. Can J Psychiatry 2001 June;46 Suppl 1:38S-58S.

6. Begley CE, Annegers JF, Swann AC, et coll. The lifetime cost of bipolar disorder in the US: an estimate for new cases in 1998. Pharmacoeconomics 2001;19(5 Pt 1):483-95.
7. Greenberg PE, Kessler RC, Birnbaum HG, et coll. The economic burden of depression in the United States: how did it change between 1990 and 2000? J Clin Psychiatry 2003 December;64(12):1465-75.

8. Kessler RC, Akiskal HS, Ames M, et coll. Considering the costs of bipolar depression. Behav Healthc 2007 January;27(1):45-7.

9. Judd LL, Akiskal HS. The prevalence and disability of bipolar spectrum disorders in the US population: re-analysis of the ECA database taking into account subthreshold cases. J Affect Disord 2003 January;73(1-2):123-31.

10. Dean BB, Gerner D, Gerner RH. A systematic review evaluating health-related quality of life, work impairment, and healthcare costs and utilization in bipolar disorder. Curr Med Res Opin 2004;20(2):139-54.

11. Akiskal HS, Bourgeois ML, Angst J, et coll. Re-evaluating the prevalence of and diagnostic composition within the broad clinical spectrum of bipolar disorders. J Affect Disord 2000 September;59 Suppl 1: S5-S30.

12. Angst J, Gamma A, Benazzi F, et coll. Toward a re-definition of subthreshold bipolarity: epidemiology and proposed criteria for bipolar-II, minor bipolar disorders and hypomania. J Affect Disord 2003 January;73(1-2):133-46.

13. Judd LL, Schettler PJ, Akiskal HS, et coll. Long-term symptomatic status of bipolar I vs. bipolar II disorders. Int J Neuropsychopharmacol 2003 June;6(2):127-37.

14. Judd LL, Akiskal HS, Maser JD, et coll. A prospective 12-year study of subsyndromal and syndromal depressive symptoms in unipolar major depressive disorders. Arch Gen Psychiatry 1998 August;55(8):694-700. 
15. Judd LL, Akiskal HS. Depressive episodes and symptoms dominate the longitudinal course of bipolar disorder. Curr Psychiatry Rep 2003 December;5(6):417-8.

16. Judd LL, Akiskal HS, Schettler PJ, et coll. The long-term natural history of the weekly symptomatic status of bipolar I disorder. Arch Gen Psychiatry 2002 June;59(6):530-7.

17. Judd LL, Akiskal HS, Schettler PJ, et coll. Psychosocial disability in the course of bipolar I and II disorders: a prospective, comparative, longitudinal study. Arch Gen Psychiatry 2005 December;62(12):1322-30.

18. Kessler RC, Berglund P, Demler O, et coll. The epidemiology of major depressive disorder: results from the National Comorbidity Survey Replication (NCS-R). JAMA 2003 June 18;289(23):3095-105.
19. Katon WJ. Clinical and health services relationships between major depression, depressive symptoms, and general medical illness. Biol Psychiatry 2003 August 1;54(3):216-26.

20. Lin EH, Von Korff M, Ludman EJ, et coll. Enhancing adherence to prevent depression relapse in primary care. Gen Hosp Psychiatry 2003 September;25(5):303-10.

21. Kessler RC, Rubinow DR, Holmes C, et coll. The epidemiology of DSMIII-R bipolar I disorder in a general population survey. Psychol Med 1997 September;27(5):1079-89.
22. McIntyre RS, Konarski JZ, Soczynska JK, et coll. Medical comorbidity in bipolar disorder: implications for functional outcomes and health service utilization. Psychiatr Serv 2006 August;57(8):1140-4.

23. McIntyre RS, Konarski JZ, Wilkins K, et coll. The prevalence and impact of migraine headache in bipolar disorder: results from the Canadian Community Health Survey. Headache 2006 June;46(6):973-82.

(24) McIntyre RS, Konarski JZ, Wilkins K, et coll. Obesity in Bipolar Disorder and Major Depressive Disorder: Results from a National Community Health Survey on Mental Health and Well-Being. Can J Psychiatry 2006;51:274-80. 